\title{
Caracterização do Bullying entre escolares: uma pesquisa realizada no município de Anta Gorda - RS
}

\author{
Characterization of Bullying among schoolchildren: a survey conducted in \\ the city of Anta Gorda - RS
}

Sandra Mara Mayer ${ }^{1 *}$, Letícia Borniatti ${ }^{1}$, Heloisa Elesbão $^{1}$, Leticia Borfe $^{1}$

ARTIGO ORIGINAL | ORIGINAL ARTICLE

\begin{abstract}
RESUMO
O presente estudo caracteriza-se por ser um estudo descritivo exploratório que teve como objetivo verificar o perfil dos alunos do $6^{\circ}$ ao $9^{\circ}$ ano de duas escolas da rede pública e privada no município de Anta GordaRS que são alvos do bullying, bem como identificar as formas e locais onde ocorrem as agressões. A amostra foi composta por 257 alunos, com idade entre 11 e 17 anos, estudantes de duas escolas do município de Anta Gorda- RS. Para a coleta de resultados foi utilizado um questionário, composto por quatro blocos, o primeiro bloco contém perguntas que visam identificar a idade, sexo, ano que estuda. Nos blocos restantes encontram-se perguntas com alternativas, direcionadas ao bullying, elas questionam se o sujeito já sofreu bullying, como ocorreu e o local da agressão. A análise estatística foi realizada através do programa SPSS v. 23.0, considerando um nível de significância de $\mathrm{p}<0.05$. Os resultados indicaram frequência significativamente maior $(\mathrm{p}=0.049)$ de agressões na escola pública, tendo como principal forma de agressão a verbal. O local em que ocorreram as agressões diferiu entre as escolas. O presente estudo identificou índice consideravelmente alto de alunos que já sofreram bullying, principalmente na rede pública. Palavras-chave: bullying na escola, agressividade, vítimas.
\end{abstract}

\begin{abstract}
The present study is characterized by being an exploratory descriptive study that aimed to verify the profile of 6th to 9th grade students from two public and private schools in the city of Anta Gorda, RS, which are targets of bullying, as well as identify the forms and places where the aggressions occur. The sample consisted of 257 students, aged between 11 and 17 years, students from two schools in the city of Anta Gorda, RS. For the collection of results was used a questionnaire, composed of four blocks, the first block contains questions that aim to identify the age, sex, year studied. In the remaining blocks are questions with alternatives, directed to the bullying, they question if the subject has already suffered bullying, as it happened and the place of the aggression. Statistical analysis was performed through the SPSS v. 23.0, considering a level of significance of $\mathrm{p}<0.05$. The results indicated a significantly higher frequency $(\mathrm{p}=$ 0.049) of aggressions in the public school, having as main form of verbal aggression. The place where the aggressions took place differed between schools. The present study identified a very high rate of students who have already suffered bullying.

Keywords: bullying at school, aggression, victims.
\end{abstract}

\footnotetext{
${ }^{1}$ Universidade de Santa Cruz do Sul, Rio Grande do Sul, Brasil

* Autor correspondente: smmayer@unisc.br
} 


\section{INTRODUÇÃO}

Bullying, apesar de ser um termo antigo, vem se disseminando e tornando-se cada vez mais presente (Alves, 2016). É uma palavra de origem inglesa, que nos reporta a um sujeito violento, grosseiro, valente e brigão. Esse termo é utilizado para denominar de maneira ampla, variadas atitudes de caráter violento, em que seja demonstrada indisciplina, humilhações, deboches e insultos (Azevedo, Gurpilhares, Ferreira \& Costa, 2014).

É preciso compreender que o bullying refere-se a atos agressivos que ocorrem de maneira repetitiva e intencional (Almeida, Cardoso \& Costac, 2009), visando unicamente humilhar, causar medo e ferir o próximo (Sales \& Sousa, 2012). É um problema frequente, encontrado em variados tipos de instituições e redes de ensino (Almeida et al., 2009).

O bullying pode ocorrer de variadas formas, as agressões poderão ser de caráter físico, verbal, moral, psicológico, sexual ou material, podendo ocorrer ainda o cyberbullying, que é a ocorrendo do bullying através da internet (Klein, 2011). Cabe ressaltar que as consequências da prática do bullying, afetam negativamente tanto as vítimas quanto os agressores (Dijk, Poorthuis \& Malti, 2017).

É preciso que a comunidade escolar pense e reflita, com o intuito de encontrar um caminho a se seguir, visando a conscientização de todos sobre as consequências negativas que o bullying traz as pessoas que se envolvem a ele, em que a principal forma de se realizar essa conscientização se dá pela educação, buscando uma união de todos os setores da escola para juntos caminharem em prol de uma solução para o problema (Elesbão, Borfe \& Mayer, 2016). Com base nisso, vê-se a importância do desenvolvimento de trabalhos como este, com o intuito de colaborar com a criação de conhecimentos pertinentes a área da Educação Física Escolar.

O presente estudo tem o objetivo verificar o perfil dos alunos do $6^{\circ}$ ao $9^{\circ}$ ano de duas escolas da rede pública e privada no município de Anta Gorda- RS que são alvos do bullying, bem como identificar as formas e locais onde ocorrem as agressões.

\section{MÉTODO}

Está pesquisa trata-se de uma pesquisa descritiva-exploratória, que é definida por Campos (2001) como uma pesquisa que visa interpretar e conhecer a realidade, por meio de uma descrição, fazendo uso de bibliografia, entrevista, entre outros.

\section{Participantes}

Os sujeitos deste estudo são 257 adolescentes, de ambos os sexos, com a faixa etária entre $11 \mathrm{e}$ 17 anos, estudantes de duas escolas do município de Anta Gorda - RS, uma da rede privada e outra da rede pública. Destes, 32 escolares foram excluídos do estudo, pois ao deixarem o questionário incompleto foram enquadrados no critério de exclusão, sendo assim a amostra final foi composta por 225 escolares.

O estudo foi aprovado pelo Comitê de Ética em Pesquisa com Seres Humanos (CEP), da Universidade de Santa Cruz do Sul (UNISC), sob parecer $\mathrm{n}^{\circ} 2.167 .790$. Sendo que os escolares com idade maior ou igual a 12 anos assinaram o termo de assentimento e os pais ou responsáveis pelos escolares assinaram um termo de consentimento livre e esclarecido (TCLE). Cabe ressaltar, que o estudo segue a resolução 466/12 do Conselho Nacional de Saúde (CNS).

\section{Instrumentos e Procedimentos}

Para a coleta de dados utilizou-se o questionário de Olweus (1993), adaptado por Mayer (2000). O questionário era composto por quatro blocos, o primeiro bloco difere dos demais, pois contém perguntas que visam identificar a idade, sexo, ano que estuda. Nos blocos restantes encontram-se perguntas com alternativas, direcionadas ao bullying, elas questionam se o sujeito já sofreu bullying, como essa agressão ocorreu, bem como o local da agressão.

\section{Análise estatística}

A análise estatística foi realizada por meio do programa SPSS v. 23.0 (IBM, Armonk, NY, EUA). Os dados foram apresentados por meio da estatística descritiva, através da frequência absoluta e relativa. Para análise do nível de 
significância foi realizado o teste qui-quadrado, sendo considerando o valor $\mathrm{p}<0.05$.

\section{RESULTADOS}

Na Tabela 1 apresenta-se a caracterização dos sujeitos. Observa-se que em ambas as escolas o maior número de alunos se encontra no $6^{\circ}$ ano, além disso, a maioria dos alunos é do sexo masculino e possui idade entre 13 e 14 anos.

Ao se analisar a tabela 2, observa-se que a ocorrência de agressões na escola pública $(46,70 \%)$ foi significantemente superior $(\mathrm{p}=0,049)$ a escola privada $(35,00 \%)$. A forma de agressão mais relatada em ambas as escolas foi a verbal, "me disseram nomes feios/disseram coisas de mim ou do meu corpo", porém ocorrendo de maneira mais expressiva na escola pública $(p=0,045)$. Quanto ao local da ocorrência das agressões, pode-se verificar que houve diferença entre as escolas $(p=0,024)$, sendo que na escola pública o local em que mais ocorreram as agressões foi a sala de aula $(25,40 \%)$ e na privada o recreio $(18,40 \%)$.

Tabela 1

Caracterização dos participantes

\begin{tabular}{|c|c|c|c|c|c|}
\hline & \multicolumn{2}{|c|}{ Privada } & \multicolumn{2}{|c|}{ Pública } & \multirow[b]{2}{*}{$\mathrm{p}$} \\
\hline & $\mathrm{n}=103$ & $(\%)$ & $\mathrm{n}=122$ & $(\%)$ & \\
\hline & \multicolumn{5}{|c|}{ Ano } \\
\hline $6^{\circ}$ ano & 32 & (31.10) & 40 & $(32.80)$ & \multirow{4}{*}{0.322} \\
\hline $7^{\circ}$ ano & 26 & $(25.20)$ & 34 & (27.90) & \\
\hline $8^{\circ}$ ano & 21 & (20.40) & 31 & (25.40) & \\
\hline $9^{\circ}$ ano & 24 & $(23.30)$ & 17 & (13.90) & \\
\hline \multicolumn{6}{|l|}{ Sexo } \\
\hline Menino & 54 & (52.40) & 65 & (53.30) & \multirow[t]{2}{*}{0.503} \\
\hline Menina & 49 & $(47.60)$ & 57 & $(46.70)$ & \\
\hline \multicolumn{6}{|l|}{ Faixa etária } \\
\hline $11-12$ anos & 44 & $(42.70)$ & 41 & $(33.60)$ & \multirow{3}{*}{0.309} \\
\hline $13-14$ anos & 45 & $(43.60)$ & 54 & (44.30) & \\
\hline $15-17$ anos & 14 & $(13.60)$ & 27 & $(22.10)$ & \\
\hline
\end{tabular}

Tabela 2

Ocorrência, forma e lugar em que mais ocorreram as agressões

\begin{tabular}{lccccc} 
& \multicolumn{3}{c}{ Privada } & \multicolumn{2}{c}{ Pública } \\
& $\mathrm{n}=103$ & $(\%)$ & $\mathrm{n}=122$ & $(\%)$ & $\mathrm{p}$ \\
\hline Você já foi agredido alguma vez na escola? & & & & & \\
Sim & 36 & $(35.00)$ & 57 & $(46.70)$ & 0.049 \\
Não & 67 & $(65.00)$ & 65 & $(53.30)$ & \\
Como te agrediram? & & & & & \\
$\quad$ Ninguém se meteu comigo & 67 & $(65.00)$ & 65 & $(5330)$ \\
Me bateram, me deram socos e pontapés ou chutes & 4 & $(3.90)$ & 3 & $(2.50)$ & $(4.90)$ \\
Me roubaram coisas & 1 & $(1.00)$ & 6 & $(4.10)$ & 0.045 \\
Me causaram medo & 3 & $(2.90)$ & 5 & $(30.30)$ \\
Me disseram nomes feios. Disseram coisas de mim ou do meu corpo & 16 & $(15.50)$ & 37 & $(30.30)$ \\
Falaram de mim, contaram segredos meus & 8 & $(7.80)$ & 4 & $(3.30)$ \\
Não falaram comigo & 2 & $(1.90)$ & 2 & $(1.60)$ \\
Me fizeram outras coisas. & 2 & $(1.90)$ & 0 & $(0.00)$ \\
Quando é que te agrediram (lugar)? & & & & \\
Ninguém me agrediu & 67 & $(65.00)$ & 65 & $(53.30)$ \\
No recreio & 19 & $(18.40)$ & 15 & $(12.30)$ \\
Na cozinha/bar & 0 & $(0.00)$ & 0 & $(0.00)$ & 0.024 \\
Nos corredores e nas escadas & 2 & $(1.90)$ & 7 & $(5.70)$ \\
Nas salas de aula & 11 & $(10.70)$ & 31 & $(25.40)$ \\
Em outro lugar & 4 & $(3.90)$ & 4 & $(3.30)$ \\
\hline
\end{tabular}

\section{DISCUSSÃO}

O presente estudo buscou verificar o perfil dos do $6^{\circ}$ ao $9^{\circ}$ ano da rede de ensino pública e privada no município de Anta Gorda- RS que são alvos do bullying, bem como identificar as formas e locais onde ocorrem as agressões, realizando um comparativo entre as duas escolas que participaram desse estudo, uma pública e outra privada. Ao se observar os resultados encontrados, identifica-se que $46,70 \%$ dos alunos 
da escola da rede pública sofreram algum tipo de agressão, já na escola privada este índice é menor $(35,00 \%)$, esses dados divergem de um estudo realizado por Aguiar e Barrera (2017) no interior do estado de São Paulo, os autores encontraram um maior índice de agressão na escola privada (78,00\%) do que na pública $(60,00 \%)$, esses índices além de divergirem, são maiores dos encontrados neste estudo. Em um estudo realizado em Lavras - MG, Araújo e Reis (2012) identificaram que $42,00 \%$ dos alunos já haviam sofrido agressões na escola. Já, em um estudo realizado por Malhi, Bharti e Sidhu (2014), no norte da Índia, 19,20\% dos alunos já sofreram agressões na escola. Por fim, em um estudo realizado em Portugal por Carvalhosa, Lima e Matos (2001) detectou-se que $47,4 \%$ dos alunos já haviam sido agredidos.

Em relação a maneira como foram agredidos, os alunos mencionam a agressão verbal (15,5\%) na escola privada e $30,3 \%$ na escola pública) principalmente, assinalando a opção "me disseram nomes feios, disseram coisas de mim ou do meu corpo". Em um estudo realizado por Rocha (2011) em Natal - RN 59,45\% dos alunos relataram a agressão verbal como a maior forma de agressão sofrida, sendo está a segunda forma de agressão mais citada em um estudo realizado por Paredes, Sanabria-Ferrand, GonzálezQuevedo e Rehalpe, (2010) na Colômbia (7,07\%). Já em um estudo realizado por Martins (2005) em uma cidade do sudeste de Portugal, com escolas de ensino médio e secundário, a principal forma de agressão relatada foi "falar mal do colega" com (67\%), que se equivale a agressão verbal. Um estudo realizado no país de Basco, por Machimbarrena e Garaigordobil (2017), apontou diferenças entre as escolas da rede pública e privada, no que diz respeito a frequência das agressões, os dados apontam que escolas públicas possuem um maior índice de violência ao serem comparadas as da rede privada, dados estes que vão ao encontro dos achados em nosso estudo.

No que diz respeito ao local em que mais ocorreram as agressões, $18,4 \%$ dos alunos da escola privada relataram acontecer durante $\mathrm{o}$ recreio, enquanto na escola pública $25,4 \%$ relataram acontecer dentro das salas de aula. No estudo realizado por Francisco e Libório (2009) na cidade de Presidente Prudente - SP constatouse que os locais com maiores índices de agressões são as salas de aula e o recreio. Além disso, os resultados de nosso estudo vão ao encontro dos encontrados no estudo de Aguiar e Barrera (2017). Os dados da escola privada são confirmados pelo estudo de Pereira, Silva e Nunes (2009), em que se apresentou o recreio escolar como local principal de ocorrências das agressões.

\section{CONCLUSÕES}

Pode-se verificar que no presente estudo encontraram-se índices consideravelmente elevados de alunos que sofreram algum tipo de agressão na escola, essa frequência se torna ainda mais elevada ao considerarmos os valores encontrados na escola da rede pública. Dentre as formas de agressões citadas, a verbal foi a mais citada nas duas escolas, sendo novamente mais elevada na escola da rede pública. O fator em que houve diferença, entre as escolas, foi quanto ao local das agressões, em que foi citada a sala de aula como principal local na escola da rede pública e o recreio na escola privada.

Conclui-se, portanto, que apesar de terem sidos encontrados resultados mais expressivos na escola da rede pública, a escola da rede privada também apresentou índices elevados de ocorrência do bullying.

Agradecimentos:

As escolas participantes da pesquisa, por terem colaborado com o desenvolvimento do estudo.

\section{Conflito de Interesses:}

Nada a declarar.

\section{Financiamento:}

Nada a declarar.

\section{REFERÊNCIAS}

Aguiar, L. G. F., \& Barrera, S. D. (2017). Manifestações de bullying em diferentes contextos escolares: um estudo exploratório. Psicologia: Ciência e Profissão, 37(3), 669-682.

Almeida, S. B. de., Cardoso, L. R. D., \& Costac, V. V. (2009). Bullying: Knowledge and practices of pedagogy in school environment. Psicologia Argumentativa, 27(58), 201-206. 
Araújo, R. S., \& Reis, F. P. G. (2012). Avaliação diagnóstica sobre a ocorrência de bullying em duas escolas estaduais de Lavras, MG: perspectivas para o planejamento em Educação Física escolar. Lecturas: Educación Física y Deportes, 17(167), 1-6.

Azevedo, L. M. R., Gurpilhares, M. S. S., Ferreira, F. S., \& Costa, W. (2014). Bullying na escola: um sofrimento. Educação, Cultura e Comunicação, 5(10), 7-20.

Campos, L. F. (2001). Métodos de Técnicas de Pesquisa. São Paulo: Alínea.

Carvalhosa, S. F. De., Lima, L., \& Matos, M. G. De. (2001). Bullying - A provocação/vitimação entre pares no contexto escolar português. Análise Psicológica, 18(4), 523-537.

Dijk, A. V., Poorthuis, A. M. G., \& Malti, T. (2017). Psychological processes in Young bullies versus bully-victims. Agressive Behavior, 9999, 1-10.

Elesbão, H., Borfe, L., \& Mayer, S. M. (2016). Bullying no contexto escolar: manifestação de atos agressivos na escola. Uma pesquisa realizada no município de Novo Cabrais, RS, Lecturas: Educación Física y Deportes, 20(214), 1-5.

Francisco, M. V., \& Libório, R. M. C. (2009). Um estudo sobre bullying entre escolares do ensino fundamental. Psicologia: Reflexão e crítica, 22(2), 200-207.

Klein, C. (2011). Bullying na escola: orientação aos pais e professores. ( $1^{\mathrm{a}}$ ed.). Blumenau: Blu.

Machimbarrena, J. M., \& Garaigordobil, M. (2017). Bullying/Cyberbullying in 5th and 6th grade: differences between public and private schools. Anales de Psicología, 33(2), 319-326.
Malhi, P., Bharti, B., \& Sidhu, M. (2014). Aggression in schools: psychosocial outcomes of bullying among Indian adolescentes. The Indian Journal of Pediatrics, 81 (11), 1171-1176.

Martins, M. J. D. (2005). Agressão e vitimação entre adolescentes, em contexto escolar: um estudo empírico. Análise Psicológica, 23(4), 401-425.

Mayer, S. M. Comportamento Agressivo em Escolares de $1^{\circ}$ a $8^{\circ}$ série do Ensino Fundamental de Santa Cruz do Sul: uma abordagem através da Teoria dos Sistemas Ecológicos. 2000. 114f. Dissertação (Mestrado em Desenvolvimento Regional - Área Sócio Cultural) Universidade de Santa Cruz do Sul, Santa Cruz do Sul.

Olweus, D. (1993). Bullying at school. Oxford e Cambridge: Blackwell.

Paredes, L., Sanabria-Ferrand, P. A., GonzálezQuevedo, L. A., \& Rehalpe, S. P. M. (2010). "Bullying" en las facultades de medicina colombianas, mito o realidade. Revista de la Facultad de Medicina, 18(2), 161-172.

Pereira, B., Silva, M. I., \& Nunes, B. (2009). Describe the bullying at school: study of a group of schools in the interior of Portugal. Revista Diálogo Educacional. 9(28), 455-466.

Rocha, K. de M. M. da. Atuação da escola frente à violência: estudo comparativo entre duas instituições de ensino. 2011. 177f. Dissertação (Mestrado em Assistência à Saúde) - Universidade Federal do Rio Grande do Norte, Natal.

Sales, M. P. D., \& Sousa, C. E. B. (2012). A manifestação da violência no espaço escolar. Estação Científica, 2(2), 55-64. quando especificado em contrário e nos conteúdos retirados de outras fontes bibliográficas. 